\title{
Global and pullback attractors of set-valued skew product flows
}

\author{
T. Caraballo \\ Dpto. de Ecuaciones Diferenciales y Análisis Numérico, \\ Universidad de Sevilla, Apdo. de Correos 1160 \\ 41080 Sevilla, Spain \\ email: caraball@us.es \\ P.E. Kloeden \\ FB Mathematik, Johann Wolfgang Goethe Universität, \\ D-60054 Frankfurt am Main, Germany \\ email: kloeden@math.uni-frankfurt.de \\ and \\ P. Marín-Rubio \\ Dpto. de Ecuaciones Diferenciales y Análisis Numérico, \\ Universidad de Sevilla, Apdo. de Correos 1160 \\ 41080 Sevilla, Spain \\ email: pedro@numer.us.es
}

February 26, 2003

\begin{abstract}
We investigate the asymptotic behaviour of a general set-valued skew product flow (SVSPF), that is, a set-valued cocycle mapping (coming from a nonautonomous differential equation or inclusion) driven by another, autonomous, system. Absorptivity conditions which ensure the existence of several types of attractors for such set-valued systems are established. The topological properties of and relations between these attractors, in forward and pullback senses and their strong and weak versions are analyzed. Several illustrative examples are also provided.
\end{abstract}

Key words: set-valued skew product flow, pullback attraction, weak invariance 1991 MSC: 34D45 37B25 37B75 58C06

\section{Introduction}

Set-valued analysis and attainability set functions are used to handle problems arising from differential equations without uniqueness, differential inclusions, or problems arising in control theory, viability theory, finances and economics among 
others, and have been widely studied by several authors in the last decades (cf. $[3,20,19,24,13,14]$ among others). The investigation of the asymptotic behaviour of these phenomena, global, strong or weak stability and attraction properties, needs the concept of pullback attractors when non-autonomous equations are considered, in particular stochastic and random ones which are intrinsically non-autonomous (cf. $[11,10,21,15]$ and $[16,5]$ for the weak pullback case).

However, the non-autonomous case can also be viewed within the framework of skew-product flows, which allows us to transform the problem into an autonomous one (cf. $[22,6,7,9,17,8])$ and to apply the classical theory for autonomous systems in a different, extended, phase space. On the other hand, if such an attractor exists, then we can recover the dynamics in the original phase space $\mathbb{R}^{d}$ and its asymptotic properties by using suitable projections, which is important as this original phase space is often the one that is of interest or meaningful in modelling.

The paper is organized as follows: In Section 2, we recall the concepts of skewproduct flows coming from a cocycle set-valued mapping with a related driving system. Conditions for existence of strong and weak attractors for a autonomous set-valued semidynamical system in an abstract metric space and its properties are analyzed in Section 3. Then, in Section 4 we apply these results to the case of a skew-product flow as introduced above, distinguishing between strong and weak cases. Here, the sectorial components in the original phase space $\mathbb{R}^{d}$ of the attractor in the extended phase space (under several suitable conditions) will be analyzed,

namely, for strong and weak asymptotic concepts as well as for their connections with the underlying non-autonomous semi-flow. The formalism used here allows us to obtain several different conclusions from precedent works $([4,5])$.

We also illustrate our theory with some examples and, for the sake of clarity, give most of the proofs at the end of the paper.

\section{Set-valued skew product flows}

Hereafter, we will use the following notation: $\mathcal{P}(X)$ and $\mathcal{K}(X)$ for the set of nonempty and nonempty compact subsets of a given space $X$, respectively; $H^{*}$ for the Hausdorff semi-distance, $H^{*}(A, B)=\sup _{a \in A} \operatorname{dist}(a, B)$, and $H$ for the Hausdorff distance, $H(A, B)=\max \left(H^{*}(A, B), H^{*}(B, A)\right)$.

To establish an appropriate framework for our analysis, we consider given an autonomous driving system, $\theta: \mathbb{R} \times P \rightarrow P$, where $P$ is a metric space, i.e. a group of homeomorphisms under composition on $P$ with the properties

i) $\theta_{0} p=p$ for all $p \in P$,

ii) $\theta_{t+s} p=\theta_{t} \theta_{s} p$ for all $s, t \in \mathbb{R}$.

iii) the mapping $(t, p) \mapsto \theta_{t} p$ is continuous.

For instance, one can think of an ordinary differential system in $P=\mathbb{R}^{l}$ with an autonomous globally Lipschitz and dissipative vectorfield $g$, i.e. $p^{\prime}=g(p)$. 
A set-valued skew product flow (SVSPF for short) consists of an autonomous driving system $\theta$ on a metric space $P$ and a set-valued cocycle mapping (attainability set mapping) $\Phi: \mathbb{R}^{+} \times P \times \mathbb{R}^{d} \rightarrow \mathcal{K}\left(\mathbb{R}^{d}\right)$ satisfying the following properties:

1. Compactness $\Phi(t, p, x)$ is a nonempty compact subset of $\mathbb{R}^{d}$ for all $t \geq 0, p \in P$, $x \in \mathbb{R}^{d}$

2. Initial condition

$$
\Phi(0, p, x)=\{x\}
$$

for all $p \in P$ and $x \in \mathbb{R}^{d}$;

3. Cocycle property

$$
\Phi(t+s, p, x)=\Phi\left(t, \theta_{s} p, \Phi(s, p, x)\right)
$$

for all $t \geq 0, p \in P, x \in \mathbb{R}^{d}$;

4. Continuity in time

$$
\lim _{s \rightarrow t} H(\Phi(s, p, x), \Phi(t, p, x))=0
$$

for all $s, t \geq 0$ and all $p \in P$ and $x \in \mathbb{R}^{d}$;

5. Upper semi continuity in parameter and initial conditions

$$
\lim _{q \rightarrow p, y \rightarrow x} H^{*}(\Phi(t, q, y), \Phi(t, p, x))=0
$$

uniformly in $t \in\left[T_{0}, T_{1}\right]$ for any $0 \leq T_{0}<T_{1}<\infty$ for all $p \in P$ and $x \in \mathbb{R}^{d}$.

Remark 1. Assumptions 4 and 5 imply that $\Phi$ is globally upper semi continuous (u.s.c.), i.e. if $\left(t_{n}, p_{n}, x_{n}\right) \rightarrow(t, p, x)$ as $n \rightarrow \infty$, then $H^{*}\left(\Phi\left(t_{n}, p_{n}, x_{n}\right), \Phi(t, p, x)\right) \rightarrow$ 0. Indeed,

$$
\begin{aligned}
H^{*}\left(\Phi\left(t_{n}, p_{n}, x_{n}\right), \Phi(t, p, x)\right) \leq & H^{*}\left(\Phi\left(t_{n}, p_{n}, x_{n}\right), \Phi\left(t_{n}, p, x\right)\right) \\
& +H^{*}\left(\Phi\left(t_{n}, p, x\right), \Phi(t, p, x)\right) \rightarrow 0 \quad \text { as } n \rightarrow \infty
\end{aligned}
$$

since the first term in the right hand side goes to zero by the u.s.c. in the second and third variables uniformly in time (property 5), and the second term goes to zero by the continuity (hence u.s.c.) of $\Phi$ on its first variable (property 4).

A trajectory of a set-valued cocycle $\Phi$ is a single-valued mapping $\phi_{p}:[0, T] \rightarrow$ $\mathbb{R}^{d}$ which, for the indicated $p \in P$, satisfies

$$
\phi_{p}(t) \in \Phi\left(t-s, \theta_{s} p, \phi_{p}(s)\right) \quad \text { for all } \quad 0 \leq s \leq t \leq T .
$$

A trajectory $\phi$ is called an entire trajectory if it is defined on all of $\mathbb{R}$ and satisfies (1) for all $s \leq t$. (If necessary a particular $p \in P$, we will use the notation $p$-trajectory for the above definition).

Now let us denote

$$
\mathcal{T}_{p, x}([0, T])=\left\{\phi_{p}, \text { trajectory } \phi_{p}(0)=x\right\} .
$$

Then, we can establish the following result in a similar way as in $[3,20,13]$ :

Theorem 2. The following properties holds: 
1. $\mathcal{T}_{p, x}([0, T]) \neq \varnothing$ (there exist trajectories for all $p, x$, and $\left.T>0\right)$

2. $\mathcal{T}_{p, x}([0, T]) \subseteq C\left([0, T] ; \mathbb{R}^{d}\right)$ (continuity)

3. $\mathcal{T}_{p, x}([0, T])$ is a compact subset of $C\left([0, T] ; \mathbb{R}^{d}\right)$

4. $\mathcal{T}_{p_{n}, x_{n}}([0, T]) \rightarrow \mathcal{T}_{p, x}([0, T])\left(\right.$ in $H^{*}$ on $\left.C\left([0, T] ; \mathbb{R}^{d}\right)\right)$ as $p_{n} \rightarrow p, x_{n} \rightarrow x$.

Remark 3. It is worth noticing that Theorem 2 also holds true if we consider a Banach space $X$ instead of $\mathbb{R}^{d}$ (see the proof in Section 7).

We now consider a general autonomous set-valued semidynamical system (SVSDS for short) as in Szegö and Treccani [24], that is, a set-valued mapping $\Pi: \mathbb{R}^{+} \times Y \rightarrow$ $Y$ where $Y$ is a connected metric space satisfying suitable properties. To avoid unnecessary repetitions, such properties are the stated below for the special case in which the set $Y$ is the extended phase space $P \times \mathbb{R}^{d}$.

A particular case of an autonomous set-valued semidynamical system is be that generated by a set-valued skew product flow, namely with $\Pi(t, p, x)=\left(\theta_{t} p, \Phi(t, p, x)\right)$ and satisfying the following properties:

1. $\Pi(t, p, x)$ is nonempty and compact;

2. $\Pi(0, p, x)=\{(p, x)\}$;

3. The semigroup property:

$$
\Pi(t+s, p, x)=\Pi(t, \Pi(s, p, x)) ;
$$

4. $t \mapsto \Pi(t, p, x)$ is continuous in the Hausdorff metric, for all $p, x$, i.e.

$$
H_{P \times \mathbb{R}^{d}}(\Pi(s, p, x), \Pi(t, p, x)) \rightarrow 0, \text { as } s \rightarrow t
$$

5. $(p, x) \mapsto \Pi(t, p, x)$ is upper semicontinuous in the $H^{*}$ sense, i.e.

$$
H_{P \times \mathbb{R}^{d}}^{*}(\Pi(t, q, y), \Pi(t, p, x)) \rightarrow 0, \text { as } q \rightarrow p, y \rightarrow x,
$$

uniformly in compact intervals $t \in\left[T_{1}, T_{2}\right]$.

A trajectory for an SVSDS is a single-valued mapping $\pi:[0, T] \rightarrow Y$ with $\pi(t) \in \Pi(t-s, \pi(s))$ for all $0 \leq s \leq t \leq T$. Analogously, a trajectory (or $p$ trajectory) for a SVSPF is a single-valued mapping $\pi_{p}:[0, T] \rightarrow P \times \mathbb{R}^{d}$ with $\pi_{p}(t) \in \Pi\left(t-s, \pi_{p}(s)\right)$ for all $0 \leq s \leq t \leq T$ and the first component of $\pi_{p}(0)$ equal to $p$.

Proposition 4. $\pi_{p}$ is a trajectory for the SVSPF if and only if there exists a trajectory $\phi_{p}$ of $\Phi$ such that

$$
\pi_{p}(t)=\left(\theta_{t} p, \phi_{p}(t)\right) \quad \forall t \in[0, T]
$$

The result holds true for any trajectory defined in any interval of time, and also for entire trajectories. 
Remark 5. An analogous result to Theorem 2 holds for an SVSDS $\Pi$ (and therefore for an SVSPF), replacing $C\left([0, T] ; \mathbb{R}^{d}\right)$ by $C([0, T] ; P \times X)$ in all the statements, actually, in any time interval not necessarily in the positive half line.

This is straightforward since the multi-valued mapping $F:\left\{\left(t, t_{0}\right): t \geq t_{0}\right\} \times$ $P \times X \rightarrow \mathcal{P}(P \times X)$ defined by $F\left(t, t_{0}, p, x\right):=\Pi\left(t-t_{0},(p, x)\right)$ satisfies the required conditions, since $\theta$ and $\Phi$ do (cf. [3, 20, 13, 5]).

\section{Attractors of SVSDS}

Now we consider a SVSDS $\Pi: \mathbb{R}^{+} \times Y \rightarrow \mathcal{P}(Y)$ and recall the basic concepts on attractors, which we will be apply in the following section to our skew product formulation, denoting then the extended phase space space $P \times \mathbb{R}^{d}$ by $Y$.

For the sake of clarity, subscripts $s$ and $w$ on attractors will denote strong and weak concepts.

Definition 6. A strong global attractor for an SVSDS $\Pi$ is a nonempty compact subset $\mathcal{A}_{s} \subset Y$ satisfying

1. strong invariance: $\Pi\left(t, \mathcal{A}_{s}\right)=\mathcal{A}_{s}$ for all $t \geq 0$

2. strong attraction: for every nonempty bounded subset $D$ of $Y$,

$$
\operatorname{dist}_{Y}\left(\Pi(t, D), \mathcal{A}_{s}\right) \rightarrow 0, \text { as } t \rightarrow \infty
$$

A weak global attractor for an SVSDS $\Pi$ is a nonempty compact subset $\mathcal{A}_{w} \subset Y$ satisfying

1. weak invariance: $\forall y \in \mathcal{A}_{w}$ there exists an entire trajectory $\pi: \mathbb{R} \rightarrow Y$ with $\pi(0)=y$ and $\pi(t) \in \mathcal{A}_{w}$ for all $t \in \mathbb{R}$

2. weak attraction: for every nonempty bounded subset $D$ of $Y$ and $y_{n} \in D$, there exist trajectories $\pi_{n}: \mathbb{R}^{+} \rightarrow Y$ and numbers $\tau_{n} \rightarrow \infty$ with $\pi_{n}(0)=y_{n}$ and

$$
\operatorname{dist}_{Y}\left(\pi_{n}\left(\tau_{n}\right), \mathcal{A}_{w}\right) \rightarrow 0, \text { as } n \rightarrow \infty
$$

For completeness, we recall some results ensuring the existence of such attractors.

\subsection{Strong global attractor of SVSDS}

The most simple case of an autonomous semi-flow with a compact absorbing set $\mathcal{B}_{s}$ (i.e. for every bounded set $D$ there exists $t_{D} \geq 0$ such that $\Pi(t, D) \subset \mathcal{B}_{s}$ for all $\left.t \geq t_{D}\right)$ is well known. Assume that $\mathcal{B}_{s}$ is a nonempty compact absorbing set in $Y$. Without loss of generality, we can assume that $\mathcal{B}_{s}$ is $\Pi$-positively invariant (i.e. $\Pi\left(t, \mathcal{B}_{s}\right) \subset \mathcal{B}_{s}$, for all $\left.t \geq 0\right)$. Define

$$
\mathcal{A}_{s}=\bigcap_{t \geq 0} \Pi\left(t, \mathcal{B}_{s}\right)
$$

Then, $a \in \mathcal{A}_{s}$ iff $a \in \mathcal{B}_{s}$ and there exist $\tau_{n} \rightarrow \infty, a_{n} \in \Pi\left(\tau_{n}, \mathcal{B}_{s}\right)$ with $a_{n} \rightarrow a$ as $n \rightarrow \infty$. 
Proposition 7. The set $\mathcal{A}_{s}$ has the following properties:

1. It is nonempty and compact and attracts bounded sets.

2. It is $\Pi$-invariant, therefore it is a global strong attractor. Actually it is the maximal invariant compact set, and also the minimal closed set that attracts bounded sets.

3. If $\Pi(t, x)$ is connected for all $(t, x) \in \mathbb{R}^{+} \times Y$, then $\mathcal{A}_{s}$ is also connected.

Remark 8. The existence of an absorbing set for the construction of the attractor and its properties can be relaxed to that of an attracting set (see [18, Th.1]), which can be more appropriate in some other situations (e.g. hyperbolic systems).

\subsection{Weak attractors of SVSDS}

We consider now an SVSDS $\Pi(t, x)$ and establish existence of weak attractors. To this end, we introduce the concept of a weak absorbing set $\mathcal{B}_{w}$ which is a nonempty, compact set which in addition is

- weakly positively invariant: for all $b \in \mathcal{B}_{w}$ there exists at least a trajectory $\pi$ with $\pi(0)=b$ and $\pi(t) \in \mathcal{B}_{w}$ for all $t \geq 0$.

- weakly absorbing: for all compact subset $D$ there exists $T_{D} \geq 0$ such that for any $d \in D$ there exists a trajectory $\pi$ with $\pi(0)=d$ and $\pi(t) \in \mathcal{B}_{w}$ for all $t \geq T_{D}$.

Theorem 9. Assume there exists a weak absorbing set $\mathcal{B}_{w}$ for an SVSDS П. Then, there exists the maximal weak attractor $\mathcal{A}_{w}$ w.r.t. $\mathcal{B}_{w}$, which is defined as the set of points $a \in \mathcal{B}_{w}$ such that there exist $b_{n} \in \mathcal{B}_{w}, \tau_{n} \rightarrow \infty$ and trajectories $\pi_{n}: \mathbb{R}^{+} \rightarrow \mathcal{B}_{w}$ with $\pi_{n}(0)=b_{n}$ and $\operatorname{dist}\left(\pi_{n}\left(\tau_{n}\right), a\right) \rightarrow 0$ as $n \rightarrow \infty$.

Remark 10. As in [5, lemma 13], we observe that an entire trajectory $\pi: \mathbb{R} \rightarrow Y$ satisfies that

$$
\pi(t) \in \mathcal{B}_{w} \quad \text { if and only if } \quad \pi(t) \in \mathcal{A}_{w} .
$$

Therefore, $\mathcal{A}_{w}$ becomes the set of points reached by entire trajectories contained in $\mathcal{B}_{w}$.

\section{Attractors of SVSPF}

Now specialize to $\Pi=(\theta, \Phi)$ with our skew product structure, i.e. we consider now that $Y=P \times \mathbb{R}^{d}$. We again split our analysis into two cases concerning the strong and weak situations. 


\section{1 (Strong) Global Attractor of SVSPF}

Suppose that there exists a compact positive invariant absorbing set $\mathcal{B}_{s} \subset P \times \mathbb{R}^{d}$ for $\Pi$. Then, there exists a strong global attractor

$$
\mathcal{A}_{s}=\bigcap_{t \geq 0} \Pi\left(t, \mathcal{B}_{s}\right)
$$

Let $P^{*}=\operatorname{Pr}_{P}\left(\mathcal{A}_{s}\right)$ be the projection of $\mathcal{A}_{s}$ onto the space $P$ and consider the decomposed notation

$$
\mathcal{A}_{s}=\bigcup_{p \in P^{*}}\{p\} \times A_{s}(p) .
$$

Proposition 11. Under the previous assumptions the following properties hold:

1. $P^{*}$ is nonempty, compact and $\theta_{t} P^{*}=P^{*}$. In fact, $P^{*}$ is the global attractor of the (single-valued) autonomous driving system $\theta$ on $P$.

2. $A_{s}\left(p^{*}\right)$ is nonempty and compact for each $p^{*} \in P^{*}$. It also satisfies the invariance property $A_{s}\left(\theta_{t} p^{*}\right)=\Phi\left(t, p^{*}, A_{s}\left(p^{*}\right)\right)$.

3. The mapping $P^{*} \ni p \mapsto A_{s}(p)$ is upper semi continuous.

Now consider the restriction $\Pi^{*}$ of $\Pi$ to $P^{*} \times \mathbb{R}^{d}$. Since $\theta_{t} P^{*}=P^{*}$ for all $t \in \mathbb{R}$, it follows that $\Pi^{*}$ is an SVSPF on $P^{*} \times \mathbb{R}^{d}$.

Consider $\mathcal{B}_{s}^{*}=\mathcal{B}_{s} \cap\left(P^{*} \times \mathbb{R}^{d}\right)$. Then, $\mathcal{B}_{s}^{*}$ absorbs sets in $P^{*} \times \mathbb{R}^{d}$ under $\Pi^{*}(\equiv \Pi)$. Also $\mathcal{B}_{s}^{*}$ is nonempty, compact and $\Pi^{*}$-positively invariant, so $\Pi^{*}$ has a maximal global attractor

$$
\mathcal{A}_{s}^{*}=\bigcup_{p \in P^{*}}\{p\} \times A_{s}^{*}(p)
$$

in $P^{*} \times \mathbb{R}^{d}$. Then, we have the following result:

Proposition 12. The strong global attractors of $\Pi$ and $\Pi^{*}$ coincide: $\mathcal{A}_{s}^{*} \equiv \mathcal{A}_{s}$.

\subsection{Weak Attractors of SVSPF}

Suppose that an SVSPF $\Pi$ has a weak attractor relative to the compact weak absorbing set $\mathcal{B}_{w} \subset P \times \mathbb{R}^{d}$, which is also given by

$$
\mathcal{A}_{w}=\bigcup_{p \in P^{*}}\{p\} \times A_{w}(p)
$$

(again we denote $P^{*}=\operatorname{Pr}_{P} \mathcal{A}_{w}$ ) as described above for general set-valued semi dynamical systems. Then, we have

Proposition 13. The following properties hold:

1. $P^{*}$ is the strong global attractor for the driving system.

2. $A_{w}(p)$ is a nonempty compact set for each $p \in P^{*}$. 
3. The map $P^{*} \ni p \mapsto A_{w}(p) \in \mathcal{K}\left(\mathbb{R}^{d}\right)$ is u.s.c.

4. $\mathcal{A}_{w}$ is weakly invariant, i.e. if $(p, a) \in \mathcal{A}_{w}$, there exists an entire trajectory $\pi=\left(\theta, \varphi_{p}\right)$ such that $\varphi_{p}(0)=a$ and $\pi(t)=\left(\theta_{t} p, \varphi_{p}(t)\right) \in \mathcal{A}_{w}$ for all $t \in \mathbb{R}$, i.e. $\varphi_{p}(t) \in A_{w}\left(\theta_{t} p\right)$.

Moreover, $\mathcal{A}_{w}$ is the largest weak invariant set in the absorbing set $\mathcal{B}_{w}$.

If we restrict $\Pi$ to $\Pi^{*}$ on $P^{*} \times \mathbb{R}^{d}$, we have that $\Pi(t,(p, x))=\Pi^{*}(t,(p, x))$ for all $t \geq 0$ and $(p, x) \in P^{*} \times \mathbb{R}^{d}$ since $P^{*}$ is $\theta$-invariant.

Define

$$
\mathcal{B}_{w}^{*}=\bigcup_{p \in P^{*}}\{p\} \times B_{w}(p) \subset \mathcal{B}_{w}=\bigcup_{p \in P}\{p\} \times B_{w}(p) .
$$

Note that $A_{w}(p) \subset B_{w}(p)$ for all $p \in P^{*}$. So, $B_{w}(p)$ is nonempty and compact. We also have that $\mathcal{B}_{w}^{*}$ is weakly positive invariant since $P^{*}$ is $\theta$-invariant and $\mathcal{B}_{w}$ is weakly positive $\Pi$-invariant.

Then, we obtain a maximal weak attractor for $\Pi^{*}, \mathcal{A}_{w}^{*}$, with respect to $\mathcal{B}_{w}^{*}$ and we will use the notation

$$
\mathcal{A}^{*}=\bigcup_{p \in P^{*}}\{p\} \times A_{w}^{*}(p) .
$$

Remark 14. Note that $a \in A_{w}^{*}(p)$ if and only if there exist sequences $t_{n} \rightarrow \infty$, $\left(p_{n}, b_{n}\right) \in \mathcal{B}_{w}^{*}$, trajectories $\pi_{n}=\left(\theta, \varphi_{p_{n}}\right)$ with $\varphi_{p_{n}}(0)=b_{n}$ and $\theta_{t_{n}} p_{n} \rightarrow p$ and $\varphi_{p_{n}}\left(t_{n}\right) \rightarrow a$.

Observe that $\mathcal{A}_{w}^{*}$ consists of entire $\Pi^{*}$-trajectories, and, since it is weak invariant, $A_{w}^{*}(p)$ is nonempty and compact for all $p \in P^{*}$.

$\mathcal{A}_{w} \subset \mathcal{B}_{w}^{*} \subset \mathcal{B}_{w}$ but $\mathcal{A}_{w}$ is the maximal $\Pi$-weak invariant family contained in $\mathcal{B}_{w}$ and $\mathcal{A}_{w}^{*}$ is $\Pi^{*}$-weak invariant (and so $\Pi$-weak invariant), therefore one has that

$$
\mathcal{A}_{w}^{*} \subset \mathcal{A}_{w} .
$$

Indeed, as $\Pi$ and $\Pi^{*}$ coincide in $\mathcal{B}_{w}^{*}$, entire $\Pi$-trajectories in $\mathcal{A}_{w}$ are $\Pi^{*}$-trajectories and conversely, by the same argument of maximality, we conclude that

$$
\mathcal{A}_{w}=\mathcal{A}_{w}^{*}
$$

Thus, we can restrict ourselves to the dynamics on $\mathcal{B}_{w}^{*}$ and we will study the relations between weak and strong skew-product attractors and their sections with respect to pullback weak and strong attractors in the following section.

\section{$5 \quad$ Pullback structure of SVSPF attractors}

Once again we split our analysis into two cases: strong and weak attractors. 


\subsection{The strong case}

As before, $\mathcal{B}_{s}$ denotes a $\Pi$-positive invariant compact absorbing set and $\mathcal{B}_{s}^{*}=$ $\mathcal{B}_{s} \cap\left(P^{*} \times \mathbb{R}^{d}\right)$ is a $\Pi^{*}$-positive invariant compact absorbing set (we keep using the notation $\mathcal{B}_{s}=\bigcup_{p \in P}\{p\} \times B_{s}(p)$, so $B_{s}(p) \neq \varnothing$ for $\left.p \in P^{*}\right)$. This implies $\Phi$-positive invariance for the sections $B_{s}(p)$ in $P^{*}$ :

$$
\Phi\left(t, p, B_{s}(p)\right) \subset B_{s}\left(\theta_{t} p\right) \quad \forall t \geq 0, \forall p \in P^{*} .
$$

Indeed, $\Pi^{*}\left(t, \mathcal{B}_{s}^{*}\right) \subset \mathcal{B}_{s}^{*}$ implies that

$$
\Pi\left(t,\left(p, B_{s}(p)\right)\right)=\left(\theta_{t} p, \Phi\left(t, p, B_{s}(p)\right)\right) \subset \mathcal{B}_{s}^{*}=\bigcup_{q \in P^{*}}\left(q, B_{s}(q)\right)
$$

what necessarily means our claim if $p \in P^{*}$.

Lemma 15. Define

$$
\hat{A}_{s}(p)=\bigcap_{t \geq 0} \Phi\left(t, \theta_{-t} p, B_{s}\left(\theta_{-t} p\right)\right) \quad \text { for } \quad p \in P^{*} .
$$

Then, $\hat{A}_{s}(p)$ is nonempty and compact for all $p \in P^{*}$.

Remark 16. If $B_{s}(p)$ and $\Phi(t, p, x)$ are connected for all $t \geq 0, p \in P^{*}$ and $x \in$ $\mathbb{R}^{d}$, then $\hat{A}_{s}(p)$ is also connected, since it is the intersection of a nested family of nonempty, compact, connected sets.

Proposition 17. The following identities hold:

$$
\hat{A}_{s}(p)=A_{s}^{*}(p)=A_{s}(p) \quad \forall p \in P^{*} .
$$

Moreover, we also have the following result.

Proposition 18. $\hat{A}_{s}(p)$ is the pullback attractor for $\Phi$ on $P^{*} \times \mathbb{R}^{d}$.

\section{Remark 19.}

(i) Notice that Propositions 17 and 18 implies that the p-components of the strong global П-attractor $\mathcal{A}_{s}$, which attracts in the forward and pullback senses, are the strong pullback attractor for $\Phi$ when the dynamics are restricteed to $P^{*} \times \mathbb{R}^{d}$.

(ii) Here we started with a global attractor for the skew-product flow $\Pi$ and have obtained a pullback attractor for $\Phi$. The converse is not true in general, i.e. if $\left\{A_{s}(p), p \in P^{*}\right\}$ is a strong pullback attractor for $\Phi$, then $\mathcal{A}=\bigcup_{p \in P^{*}}\{p\} \times$ $A_{s}(p)$ may not be a global attractor for $\Pi$ (see [8] for a counterexample in the single-valued case).

(iii) In general, pullback attractors for set-valued flows are only negatively invariant, and strict invariance needs additional assumptions (the easiest is a lower semi continuous property for the flow). Here, the strict invariance holds here since the driving system has a global attractor. 
Now, bearing (2) in mind, we deduce an improvement of the u.s.c. result in Section 4.1 when the approximation in the parameter space comes from the driving system.

Corollary 20. For every $p \in P^{*}$, the map $t \mapsto A_{s}\left(\theta_{t} p\right)$ is continuous.

Proof. It follows from the fact that $\mathcal{A}_{s}^{*} \equiv \mathcal{A}_{s}$ is strongly $\Pi$-invariant (and thus $\left.\Phi\left(t, p, A_{s}(p)\right)=A_{s}\left(\theta_{t} p\right)\right)$, and the continuity of $\Phi$ on its first variable.

\subsection{The weak case}

Assume that $\mathcal{B}_{w}=\bigcup_{p \in P}\{p\} \times B_{w}(p)$ is a weak absorbing family for the skew-product flow and denote $\mathcal{B}_{w}^{*}=\mathcal{B}_{w} \cap\left(P^{*} \times \mathbb{R}^{d}\right)$.

Then, the family of sets $\left\{B_{w}(p)\right\}_{p \in P}$ "is" (see Remark 22 below) a weak pullback absorbing family for $\Phi$, similarly to the concept of [5], which leads us to consider the following definition:

$$
\hat{A}_{w}(p)=\left\{\begin{array}{l|l}
a \in \mathbb{R}^{d} & \begin{array}{l}
\exists t_{n} \rightarrow \infty, b_{n} \in B_{w}\left(\theta_{-t_{n}} p\right), p-\text { trajectories } \\
\varphi_{n}:\left[-t_{n}, 0\right] \rightarrow \mathbb{R}^{d} \text { with } \varphi_{n}\left(-t_{n}\right)=b_{n} \text { and } \varphi_{n}(0) \rightarrow a
\end{array}
\end{array}\right\}
$$

We will now prove that $\hat{A}_{w}(p) \subset A_{w}^{*}(p)$.

Let $a \in \hat{A}_{w}(p)$, then there exist sequences $t_{n} \rightarrow \infty, b_{n} \in B_{w}\left(\theta_{-t_{n}} p\right)$, and $p$ trajectories $\hat{\varphi}_{n}:\left[-t_{n}, 0\right] \rightarrow \mathbb{R}^{d}$ such that $\hat{\varphi}_{n}\left(-t_{n}\right)=b_{n}$ and $\hat{\varphi}_{n}(0) \rightarrow a$.

Denote $p_{n}=\theta_{-t_{n}} p$ and $\varphi_{n}:\left[0, t_{n}\right] \rightarrow \mathbb{R}^{d}$ given by

$$
t \mapsto \varphi_{n}(t)=\hat{\varphi}_{n}\left(t-t_{n}\right) .
$$

It is obvious that $\pi_{n}=\left(\theta, \varphi_{n}\right)$ are $\Pi^{*}$-trajectories with $\pi_{n}(0)=\left(p_{n}, b_{n}\right)$ and

$$
\pi_{n}\left(t_{n}\right)=\left(\theta_{t_{n}} p_{n}, \varphi_{n}\left(t_{n}\right)\right)=\left(p, \hat{\varphi}_{n}(0)\right) \rightarrow(p, a) .
$$

Remark 14 implies that $\hat{A}_{w}(p) \subset A_{w}^{*}(p)$.

As for the other inclusion, we have: $\mathcal{A}_{w}^{*}$ is weakly invariant, which means that for all $(p, a) \in \mathcal{A}_{w}^{*}$, there exists an entire trajectory $\pi$ of $\Pi^{*}$ with $\pi(t) \in \mathcal{A}_{w}^{*}$ for all $t \in \mathbb{R}$, i.e. $\pi(t)=\left(\theta_{t} p, \varphi_{p}(t)\right)$ with $\varphi_{p}$ an entire trajectory of $\Phi$ such that $\varphi_{p}(0)=a \in A_{w}(p)$ and $\varphi_{p}(t) \in A_{w}\left(\theta_{t} p\right)$ for all $t \in \mathbb{R}$.

Consider any sequence $t_{n} \rightarrow \infty$ and define $p_{n}=\theta_{-t_{n}} p$. Take $b_{n}=\varphi_{p}\left(-t_{n}\right) \in$ $A_{w}\left(\theta_{-t_{n}} p\right) \subset B_{w}\left(\theta_{-t_{n}} p\right)$. Then, trivially $\varphi_{n}(t) \equiv \varphi_{p}(t)$ for all $n$ and $t$, jointly with the chosen values $b_{n}, t_{n}$ and $p_{n}$, gives that $a \in \hat{A}_{w}(p)$ and therefore $A_{w}^{*}(p) \subset \hat{A}_{w}(p)$.

Therefore, we have proved the following result:

Proposition 21. Under the above assumptions, $\hat{A}_{w}(p)=A_{w}^{*}(p)=A_{w}(p)$ for all $p \in P^{*}$, i.e. the maximal weak attractor of $\Pi$ in $\mathcal{B}_{w}$, resp. $\Pi^{*}$ in $\mathcal{B}_{w}^{*}$ give the maximal weak pullback attractor of $\Phi$ with respect to the absorbing family $\left\{B_{w}(p), p \in P^{*}\right\}$.

Remark 22. By Proposition 13 and the continuity of $\theta$, the set-valued mapping

$$
t \mapsto \hat{A}_{w}\left(\theta_{t} p\right)=A_{w}\left(\theta_{t} p\right)
$$

is (only) upper semi continuous (in comparison with [5, Prop. 11]). 


\section{Examples}

Our first example related to the strong structure of a skew product flow is given by the differential inclusion

$$
x^{\prime}(t) \in G(t, x(t))+\beta p(t, x(t))
$$

where the multi-valued mapping $G$ has good properties (for instance, it has closed and convex values in $\mathcal{P}\left(\mathbb{R}^{d}\right)$ and is Lipschitz continuous) and satisfies:

1. almost periodic dependence on its first variable,

2. a dissipativity condition: for all $y \in G(t, x)$ it holds $(x, y) \leq-\alpha_{0}|x|^{2}+\alpha_{1}$, with $\alpha_{i}>0, i=1,2$,

3. and $p$ is single-valued, almost periodic on its first variable, and satisfies

$$
\begin{gathered}
|p(t, x)| \leq \gamma_{1}(t)|x|+\gamma_{2}(t), \\
|p(t, x)-p(t, y)| \leq \gamma_{3}(t)|x-y|
\end{gathered}
$$

with $\gamma_{i}(\mathrm{i}=1,2,3)$ continuous, positive functions and

$$
\lim _{t \rightarrow \infty} \frac{1}{t} \int_{0}^{t} \gamma_{1}(s) d s=C_{f}<\infty .
$$

Under these assumptions, it is easy to deduce the existence of an attractor for the skew-product flow generated by the above problem if $\beta$ is positive and small enough. The parameter space $P$ is the product of the hulls of the mappings $G$ and $p$ in appropriate function spaces.

The almost periodic time dependence is not essential. Consider the driving system

$$
p^{\prime}(t)=g(p(t))
$$

with $g: \mathbb{R} \rightarrow \mathbb{R}$ given by

$$
g(y)=\left\{\begin{array}{lll}
-y-1 & \text { if } & y \leq-1 \\
y^{2}-1 & \text { if } & y \in(-1,1) \\
1-y & \text { if } & y \geq 1
\end{array}\right.
$$

Then, it is easy to check that the non-autonomous problem

$$
x^{\prime}(t) \in[-1,-1 / 2] x(t)+p(t)
$$

generates a set-valued cocycle mapping, and jointly with (5), a skew-product flow with a non-trivial global attractor in the strong sense.

Other examples, arising from differential equations without uniqueness, partial differential equations with periodic forcing terms, delay and functional differential models among others can be founded in $[9,6,7,8]$. 
An simple example to illustrate the weak case can be provided by using an almost periodic or even periodic function of time. Suppose we have the differential inclusion

$$
x^{\prime}(t) \in x(t)\left[-\sin ^{2} t, 0\right]
$$

which clearly generates a set-valued cocycle mapping. Since for every initial value $x_{0}$, the constant function $\phi(t)=x_{0}$ is a solution of the problem, there does not exist a strong attractor for the skew product flow. However, the set $\{0\}$ is a forward attractor for the non-autonomous system (and $[0, \pi] \times\{0\}$ is a global attractor for the skew-product flow, where $P=\mathbb{R}$ is equipped with the usual shift modulo $\pi$, which is considered as the driving operator). Indeed,

$$
\frac{x^{\prime}}{x}=-\sin ^{2} t=-1 / 2(1-\cos (2 t))
$$

generates the solutions for the initial data $x_{0}$

$$
x(t)=x_{0} e^{-t / 2+1 / 4 \sin (2 t)} .
$$

Finally, in [12] we can find some interesting examples on practical problems arising in controllability theory for linear differential systems written in the form:

$$
x^{\prime}(t)=A(t) x+B u, \quad u \in U,
$$

where $A$ is an almost periodic operator and $B$ a linear operator acting on a control set $U$. (Here $P$ is the closure of the set $\{A(t): t \in \mathbb{R}\}$ ).

\section{Proofs}

\subsection{Barbashin Theorem for set-valued cocycle maps. Proof of The- orem 2}

First, we prove a Barbashin Theorem which will be needed below. We follow the ideas in [13] to construct a trajectory. We proceed in several steps.

\section{Attainability functions}

Because of the cocycle property, for general points $x_{0}, x_{1}$, times $0 \leq t \leq t_{1}$, and $p \in P$, we have

$$
\Phi\left(t_{1}, p, x_{0}\right)=\Phi\left(t, \theta_{t_{1}-t} p, \Phi\left(t_{1}-t, p, x_{0}\right)\right) .
$$

Then, $x_{1} \in \Phi\left(t_{1}, p, x_{0}\right)$ if and only if

$$
\exists x \in \Phi\left(t_{1}-t, p, x_{0}\right) \quad \text { such that } \quad x_{1} \in \Phi\left(t, \theta_{t_{1}-t} p, x\right) .
$$

This suggests the following definition of a restricted backwards attainability function:

$$
x \in G\left(x_{0}, p ; x_{1}, t_{1} ; t\right) \Longleftrightarrow x_{1} \in \Phi\left(t, \theta_{t_{1}-t} p, x\right) .
$$

Then, one has the following result: 
Lemma 23. If $x_{1} \in \Phi\left(t_{1}, p, x_{0}\right)$, then $G\left(x_{0}, p ; x_{1}, t_{1} ; t\right)$ is nonempty and closed, and $G\left(x_{0}, p ; x_{1}, t_{1} ; 0\right)=\left\{x_{1}\right\}$.

The mapping $t \in\left[0, t_{1}\right] \rightarrow A(t) \in \mathcal{P}\left(\mathbb{R}^{N}\right)$ defined by

$$
A(t)=\Phi\left(t_{1}-t, p, x_{0}\right) \cap G\left(x_{0}, p ; x_{1}, t_{1} ; t\right)
$$

has nonempty compact images and is continuous in $t$ with respect to the Hausdorff metric.

Proof. Owing to the cocycle property, it is obvious that $G\left(x_{0}, p ; x_{1}, t_{1} ; t\right)$ is nonempty.

To prove that it is closed, we proceed as in [20]. Suppose $y_{i} \in G\left(x_{0}, p ; x_{1}, t_{1} ; t\right)$ with $y_{i} \rightarrow y$ as $i \rightarrow \infty$, then $x_{1} \in \Phi\left(t, \theta_{t_{1}-t} p, y\right)$. Indeed,

$$
d\left(x_{1}, \Phi\left(t, \theta_{t_{1}-t} p, y\right)\right) \leq d\left(x_{1}, \Phi\left(t, \theta_{t_{1}-t} p, y_{i}\right)\right)+H^{*}\left(\Phi\left(t, \theta_{t_{1}-t} p, y_{i}\right), \Phi\left(t, \theta_{t_{1}-t} p, y\right)\right) .
$$

The first term on the right hand side is zero, and the second one converges to zero due to the u.s.c. of $\Phi$, so $d\left(x_{1}, \Phi\left(t, \theta_{t_{1}-t} p, y\right)\right)=0$ and $x_{1} \in \Phi\left(t, \theta_{t_{1}-t} p, y\right)$.

The statement of $G\left(x_{0}, p ; x_{1}, t_{1} ; 0\right)=\left\{x_{1}\right\}$ is trivial.

It is clear that $A(t)$ is nonempty (see the construction of $G$ and (7)), and also compact since it is the intersection of a compact set and a closed one. We now need to prove the continuity of $t \mapsto A(t)$, i.e. we have to check that $H\left(A(s), A\left(s_{0}\right)\right) \rightarrow 0$ as $s \rightarrow s_{0}$.

We start with the case $H^{*}\left(A(s), A\left(s_{0}\right)\right) \rightarrow 0$. Suppose it is not so, i.e. there exist a constant $\varepsilon>0$ and a sequence $s_{i} \rightarrow s_{0}$, such that $H^{*}\left(A\left(s_{i}\right), A\left(s_{0}\right)\right) \geq \varepsilon$. As $A\left(s_{i}\right)$ is compact, the maximum is achieved at some point, say $z_{i}$ :

$$
H^{*}\left(A\left(s_{i}\right), A\left(s_{0}\right)\right)=d\left(z_{i}, A\left(s_{0}\right)\right) \geq \varepsilon .
$$

Since $z_{i} \in A\left(s_{i}\right) \subset \Phi\left(t_{1}-s_{i}, p, x_{0}\right)$, they belong to a compact set (by the continuity of $\Phi$ and its compact values), so there exists a convergent subsequence $z_{i^{\prime}} \rightarrow z_{0} \in \Phi\left(t_{1}-s_{0}, p, x_{0}\right)$. On the other hand, as $z_{i} \in G\left(x_{0}, p ; x_{1}, t_{1} ; s_{i}\right)$, i.e. $x_{1} \in \Phi\left(s_{i}, \theta_{t_{1}-s_{i}} p, z_{i}\right)$, because of the u.s.c. of $\Phi$ on its three variables (see Remark $1)$ and as $\left(s_{i}, \theta_{t_{1}-s_{i}} p, z_{i}\right) \rightarrow\left(s_{0}, \theta_{t_{1}-s_{0}} p, z_{0}\right)$, we have that $x_{1} \in \Phi\left(s_{0}, \theta_{t_{1}-s_{0}} p, z_{0}\right)$, i.e. $z_{0} \in G\left(x_{0}, p ; x_{1}, t_{1} ; s_{0}\right)$ and therefore $z_{0} \in A\left(s_{0}\right)$, which leads to a contradiction, since then $H^{*}\left(A\left(s_{i}\right), A\left(s_{0}\right)\right)=d\left(z_{i}, A\left(s_{0}\right)\right) \leq d\left(z_{i}, z_{0}\right) \rightarrow 0$.

We now prove that $H^{*}\left(A\left(s_{0}\right), A(s)\right) \rightarrow 0$ as $s \rightarrow s_{0}$. Arguing by contradiction, assume there exist a constant $\varepsilon>0$ and a sequence $\left\{s_{i}\right\}_{i \geq 1}$ with $s_{i} \rightarrow s_{0}$ as $i \rightarrow \infty$, such that $H^{*}\left(A\left(s_{0}\right), A\left(s_{i}\right)\right) \geq \varepsilon$. Consider $z_{i}^{0} \in A\left(s_{0}\right)$ such that $d\left(z_{i}^{0}, A\left(s_{i}\right)\right)=$ $H^{*}\left(A\left(s_{0}\right), A\left(s_{i}\right)\right)$. This is possible since $A\left(s_{0}\right)$ is compact, and indeed, w.l.o.g. we can assume that $z_{i}^{0} \rightarrow z_{0} \in A\left(s_{0}\right)$. To finish, now it is enough to prove that there exists $z_{i} \in A\left(s_{i}\right)$ such that $z_{i} \rightarrow z_{0}$.

We split into two cases (the general case is a combination of these): 
$s_{i} \leq s_{0}$ Consider

$$
z_{0} \in A\left(s_{0}\right)=\Phi\left(t_{1}-s_{0}, p, x_{0}\right) \cap G\left(x_{0}, p ; x_{1}, t_{1} ; s_{0}\right),
$$

thus $x_{1} \in \Phi\left(s_{0}, \theta_{t_{1}-s_{0}} p, z_{0}\right)$.

On the one hand, $\Phi\left(s_{0}-s_{i}, \theta_{t_{1}-s_{0}} p, z_{0}\right) \cap G\left(z_{0}, \theta_{t_{1}-s_{0}} p ; x_{1}, s_{0} ; s_{i}\right) \neq \varnothing$ by the same reasons as $A(t)$. On the other hand, one can easily see that

$$
G\left(x_{0}, p ; x_{1}, t_{1} ; s_{i}\right)=G\left(z_{0}, \theta_{t_{1}-s_{0}} p ; x_{1}, s_{0} ; s_{i}\right),
$$

and

$$
\Phi\left(s_{0}-s_{i}, \theta_{t_{1}-s_{0}} p, z_{0}\right) \subset \Phi\left(t_{1}-s_{i}, p, x_{0}\right)
$$

Therefore,

$$
\varnothing \neq \Phi\left(s_{0}-s_{i}, \theta_{t_{1}-s_{0}} p, z_{0}\right) \cap G\left(z_{0}, \theta_{t_{1}-s_{0}} p ; x_{1}, s_{0} ; s_{i}\right) \subset A\left(s_{i}\right) .
$$

Pick

$$
z_{i} \in \Phi\left(s_{0}-s_{i}, \theta_{t_{1}-s_{0}} p, z_{0}\right) \cap G\left(z_{0}, \theta_{t_{1}-s_{0}} p ; x_{1}, s_{0} ; s_{i}\right) .
$$

By the continuity of $\Phi$ we have that $z_{i} \rightarrow z_{0}$.

$s_{i} \geq s_{0}$ The same argument shows that

$$
\varnothing \neq \Phi\left(t_{1}-s_{i}, p, x_{0}\right) \cap G\left(x_{0}, p ; z_{0}, t_{1}-s_{0} ; s_{i}-s_{0}\right),
$$

and we also have the following inclusion

$$
G\left(x_{0}, p ; z_{0}, t_{1}-s_{0} ; s_{i}-s_{0}\right) \subset G\left(x_{0}, p ; x_{1}, t_{1} ; s_{i}\right)
$$

by means of the cocycle property of $\Phi$. Thus,

$$
\begin{aligned}
\varnothing & \neq \Phi\left(t_{1}-s_{i}, p, x_{0}\right) \cap G\left(x_{0}, p ; z_{0}, t_{1}-s_{0} ; s_{i}-s_{0}\right) \\
& \subset \Phi\left(t_{1}-s_{i}, p, x_{0}\right) \cap G\left(x_{0}, p ; x_{1}, t_{1} ; s_{i}\right)=A\left(s_{i}\right) .
\end{aligned}
$$

By choosing

$$
z_{i} \in \Phi\left(t_{1}-s_{i}, p, x_{0}\right) \cap G\left(x_{0}, p ; z_{0}, t_{1}-s_{0} ; s_{i}-s_{0}\right),
$$

we have that $z_{i} \in \Phi\left(\left[0, t_{1}-s_{0}\right], p, x_{0}\right)$ for all $i$, which is compact. Therefore, there exists a converging subsequence $z_{i^{\prime}} \rightarrow \xi$. On the other hand, as long as $z_{0} \in$ $\Phi\left(s_{i}-s_{0}, \theta_{t_{1}-s_{i}} p, z_{i}\right)$, particularizing to the case of the subsequence $z_{i^{\prime}}$ commented above, the u.s.c. of $\Phi$ implies $z_{0} \in \Phi\left(0, \theta_{t_{1}-s_{0}} p, \xi\right)=\{\xi\}$, so $z_{0}=\xi$ (observe that therefore the whole sequence converges), and the proof is complete.

Corollary 24. Every $\Phi$-trajectory is continuous.

Proof. Let $\phi_{p}:[0, T] \rightarrow \mathbb{R}^{d}$ be a $p$-trajectory, i.e. $\phi_{p}(t) \in \Phi\left(t-s, \theta_{s} p, \phi_{p}(s)\right)$ for all $0 \leq s \leq t \leq T$. We consider $t_{a}$ fixed, and $t \rightarrow t_{a}$. Separate the proof in two cases:

$t>t_{a}$ Then,

$$
\phi(t) \in \Phi\left(t-t_{a}, \theta_{t_{a}} p, \phi_{p}\left(t_{a}\right)\right) \rightarrow \Phi\left(0, \theta_{t_{a}} p, \phi_{p}\left(t_{a}\right)\right)=\left\{\phi_{p}\left(t_{a}\right)\right\}
$$


$t<t_{a}$ By easy computations, we see that

$$
\phi(t) \in \Phi\left(t-t_{0}, \theta_{t_{0}} p, \phi_{p}\left(t_{0}\right)\right) \cap G\left(\phi\left(t_{0}\right), \theta_{t_{0}} p, \phi\left(t_{a}\right), t_{a}-t_{0}, t_{a}-t\right) .
$$

Observe that this set has the form described in the definition (8) for $A(\tilde{t})$, but with $\tilde{x}_{0}=\phi_{p}\left(t_{0}\right), \tilde{p}=\theta_{t_{0}} p, \tilde{x}_{1}=\phi_{p}\left(t_{a}\right), \tilde{t}_{1}=t_{a}-t_{0}, \tilde{t}=t_{a}-t$. Thus, now we have that $A(\tilde{t}) \rightarrow A(0)$ as $\tilde{t}=t_{a}-t \rightarrow 0$, but $A(0)=\left\{\phi_{p}\left(t_{a}\right)\right\}$, which gives us the desired result.

\section{Proof of Theorem 2}

We prove Theorem 2 following the ideas in $[20,13]$.

In order to do that, firstly we show how to construct trajectories.

Consider $a \leq b$ and $\left(\theta_{a} p, x\right) \in P \times \mathbb{R}^{d}$. We will construct a trajectory $\phi_{p}$ with $\phi_{p}(a)=x$ passing through any other (given) point at time $b$, say $\phi_{p}(b)$, which necessarily belongs to $\Phi\left(b-a, \theta_{a} p, \phi_{p}(a)\right)$. A first step is to choose the mid time point $(a+b) / 2$ of the interval $(a, b)$ and an image for it. By the previous considerations, this image must be in

$$
\Phi\left((b-a) / 2, \theta_{a} p, \phi_{p}(a)\right) \cap G\left(\phi_{a}(a), \theta_{a} p, \phi_{p}(b), b-a,(b-a) / 2\right),
$$

which is a nonempty compact set. We iterate this process in the intervals $[a,(a+b) / 2]$ and $[(a+b) / 2, b]$. Repeating this process, we can obtain a dyadic sequence of points which satisfies the relation for being a trajectory. The problem of completing from this set to the whole interval is solved by density: let $t$ be a non dyadic point of $[a, b]$, and $t^{\prime}$ and $t^{\prime \prime}$ any dyadic points such that $t^{\prime}<t<t^{\prime \prime}$, then we choose

$$
\phi_{p}(t) \in K(t)=\bigcap_{t^{\prime}<t<t^{\prime \prime}} \Phi\left(t-t^{\prime}, \theta_{t^{\prime}} p, \phi_{p}\left(t^{\prime}\right)\right) \cap G\left(\phi_{p}\left(t^{\prime}\right), \theta_{t^{\prime}} p, \phi_{p}\left(t^{\prime \prime}\right), t^{\prime \prime}-t^{\prime}, t^{\prime \prime}-t\right) .
$$

Now, we need to check that each of these sets is nonempty (which can be seen again as in (8)), and that this construction is consistent, i.e. the finite intersection property holds. The arguments are the same as in [13].

Indeed, for any $s_{1}<s_{2}<t<s_{3}<s_{4}$, we have that the following sets are well defined and the inclusions hold:

$$
\Phi\left(\phi_{p}\left(s_{2}\right), \theta_{s_{2}} p, t-s_{2}\right) \subset \Phi\left(\phi_{p}\left(s_{1}\right), \theta_{s_{1}} p, t-s_{1}\right)
$$

and

$$
\begin{aligned}
G\left(\phi_{p}\left(s_{1}\right), \theta_{s_{1}} p ; \phi_{p}\left(s_{3}\right), s_{3}-s_{1} ; s_{3}-t\right) & =G\left(\phi_{p}\left(s_{2}\right), \theta_{s_{2}} p ; \phi_{p}\left(s_{3}\right), s_{3}-s_{2} ; s_{3}-t\right) \\
& \subset G\left(\phi_{p}\left(s_{1}\right), \theta_{s_{1}} p ; \phi_{p}\left(s_{4}\right), s_{4}-s_{1} ; s_{4}-t\right) \\
& =G\left(\phi_{p}\left(s_{2}\right), \theta_{s_{2}} p ; \phi_{p}\left(s_{4}\right), s_{4}-s_{2} ; s_{4}-t\right) .
\end{aligned}
$$

Thus, we have proved the existence of at least one trajectory. We recall that every trajectory has been proved to be continuous. 
Now we prove that $\mathcal{T}_{p, x}([0, T])$ is compact in $C\left([0, T] ; \mathbb{R}^{d}\right)$. Let be $\left\{\phi_{n}\right\} \subset$ $\mathcal{T}_{p, x}([0, T])$. As $\Phi(T, p, x)$ is compact, there exists a subsequence $\left\{\phi_{n_{1}}(T)\right\} \subset\left\{\phi_{n}(T)\right\}$ converging to a point denoted $\phi(T)$. By the same reason, there exists another subsequence $\left\{\phi_{n_{2}}(T / 2)\right\} \subset\left\{\phi_{n_{1}}(T / 2)\right\}$ converging to a point $\phi(T / 2)$ and we iterate this procedure. By a diagonal argument, we obtain a subsequence relabeled again with index $m$, converging in all the dyadic numbers of $[0, T]: \phi_{m}\left(p T / 2^{q}\right) \rightarrow \phi\left(p T / 2^{q}\right)$.

As $\left\{\phi_{n}\right\}$ are trajectories, then $\phi_{n}(t) \in \Phi\left(t-s, \theta_{s} p, \phi_{n}(s)\right)$ for all $0 \leq s \leq t \leq T$, in particular for dyadic numbers, whence $\phi(t) \in \Phi\left(t-s, \theta_{s} p, \phi(s)\right)$ by the u.s.c. of $\Phi$. To extend it to the whole interval to obtain a trajectory, we proceed as before.

Let us prove that $\phi_{n} \rightarrow \phi$ in $C\left([0, T] ; \mathbb{R}^{d}\right)$. The pointwise convergence follows easily. Indeed, for any $t$, let us write

$$
\phi_{n}(t)-\phi(t)=\phi_{n}(t)-\phi_{n}\left(t_{D}\right)+\phi_{n}\left(t_{D}\right)-\phi\left(t_{D}\right)+\phi\left(t_{D}\right)-\phi(t),
$$

with $t_{D}$ a dyadic number close enough to $t$ such that $\left|\phi\left(t^{\prime}\right)-\phi(t)\right| \leq \varepsilon / 3$, and with $H\left(\Phi(t, p, x), \Phi\left(t^{\prime}, p, x\right)\right) \leq \varepsilon / 3$. Then we can choose $n\left(t_{D}\right)$ such that for all $n \geq n\left(t_{D}\right)$, one has $\left|\phi_{n}\left(t_{D}\right)-\phi\left(t_{D}\right)\right| \leq \varepsilon / 3$. However, for the uniform convergence one needs to be more careful. We follow the proof in [20, Th.6.2]. By a contradiction argument, if it does not hold, there exist a constant $\varepsilon>0$, sequences $t_{n}$, with $t_{n} \rightarrow t \in[0, T]$, and $\phi_{n}$ such that

$$
\left|\phi_{n}\left(t_{n}\right)-\phi(t)\right|>\varepsilon \text {. }
$$

Consider a dyadic number $\tau \in\left\{\begin{array}{lll}(t, T] & \text { if } & t<T \\ \{T\} & \text { if } & t=T .\end{array}\right.$

As $\left\{\phi_{n}\left(t_{n}\right)\right\} \subset \Phi([0, T], p, x)$, which is compact, there exists a convergent subsequence (we do not relabel it) $\phi_{n}\left(t_{n}\right) \rightarrow z$. Then, there exists $n_{\tau} \in \mathbb{N}$ such that for all $n \geq n_{\tau}$, we have that $t_{n}<\tau$. Since $\phi_{n}(\tau) \in \Phi\left(\tau-t_{n}, \theta_{t_{n}} p, \phi_{n}\left(t_{n}\right)\right)$ and $\tau$ is dyadic, we have $\phi_{n}(\tau) \rightarrow \phi(\tau)$ and so, the global u.s.c. of $\Phi$ implies that

$$
\phi(\tau) \in \Phi\left(\tau-t, \theta_{t} p, z\right) .
$$

Using now the continuity of $\phi$ and the density of dyadic numbers we have:

$$
\phi(t)=\lim _{\tau \rightarrow t} \phi(\tau) \in \limsup _{\tau \rightarrow t} \Phi\left(\tau-t, \theta_{t} p, z\right)=\Phi\left(0, \theta_{t} p, z\right)=\{z\},
$$

which contradicts $(9)$.

Finally, we prove the upper semicontinuity result claimed in (4):

$$
H^{*}\left(\mathcal{T}_{p_{n}, x_{n}}([0, T]), \mathcal{T}_{p, x}([0, T]) \rightarrow 0 \quad \text { if } \quad\left(p_{n}, x_{n}\right) \rightarrow(p, x) .\right.
$$

According to the last section, it is equivalent to prove $\varepsilon$-u.s.c. We proceed again by a contradiction argument. Suppose there exist a positive constant $\varepsilon>0$, a sequence of pairs $\left(p_{n}, x_{n}\right)$ converging to $(p, x)$ in $P \times \mathbb{R}^{d}$, and trajectories $\phi_{n} \in \mathcal{T}_{p_{n}, x_{n}}([0, T])$ such that $\phi_{n} \notin B_{C\left([0, T] ; \mathbb{R}^{d}\right)}\left(\mathcal{T}_{p, x}([0, T]), \varepsilon\right)$. We will prove that for a subsequence $\phi_{n^{\prime}}$, it is satisfied that $\phi_{n^{\prime}} \rightarrow \phi \in \mathcal{T}_{p, x}([0, T])$, which will give us the contradiction. 
First, we have that $\phi_{n}(0)=x_{n} \rightarrow x$. As $\phi_{n}(T) \in \Phi\left(T, p_{n}, x_{n}\right)$, by the compact values and u.s.c. of $\Phi$, there exists a subsequence (which we do not relabel) converging to an element $\phi(T)$ in $\Phi(T, p, x)$. The same argument can be applied to this subsequence at time $T / 2$, and, taking a diagonal subsequence, for a countable set of numbers (the dyadic in $[0, T]$ ), defining a set of points $\phi\left(k T / 2^{m}\right)$. Of course, they satisfy the trajectory property, as far as $\phi_{n}$ are:

$$
\phi_{n}(t) \in \Phi\left(t-s, \theta_{s} p_{n}, \phi_{n}(s)\right),
$$

for dyadic $0 \leq s \leq t \leq T$. By the u.s.c. of $\Phi$ we have

$$
\phi(t) \in \Phi\left(t-s, \theta_{s} p, \phi(s) .\right.
$$

The extension to the whole interval is done as above, preserving the trajectory property. To finish, the uniform convergence of $\phi_{n}$ to $\phi$ is deduced as in the previous case.

\subsection{Autonomous strong attractors of SVSDS. Proof of Proposi- tion 7}

Clearly, $\mathcal{A}_{s}$ is nonempty since it is the intersection of a nested family of compact sets. Moreover, it is compact too.

Since any bounded set is absorbed by $\mathcal{B}_{s}$, it is enough to see that $\mathcal{B}_{s}$ is attracted by $\mathcal{A}_{s}$. If not, there exist $\varepsilon>0$ and a sequence $x_{n} \in \Pi\left(t_{n}, \mathcal{B}_{s}\right)$, with $t_{n} \rightarrow \infty$, such that $\operatorname{dist}\left(x_{n}, \mathcal{A}_{s}\right) \geq \varepsilon>0$. But $x_{n} \in \mathcal{B}_{s}$ for all $n \geq n\left(\mathcal{B}_{s}\right)$ by the absorbing property of $\mathcal{B}_{s}$, and as it is compact, there is a subsequence converging (we do not relabel) to $x \in \mathcal{A}_{s}$, which is a contradiction.

We check $\Pi$-invariance in two steps: First, we prove that $\Pi\left(t, \mathcal{A}_{s}\right) \subset \mathcal{A}_{s}$ as in the single-valued case. Indeed,

$$
\begin{aligned}
\Pi\left(t, \mathcal{A}_{s}\right)=\Pi\left(t, \bigcap_{r \geq 0} \Pi\left(r, \mathcal{B}_{s}\right)\right) & \subset \bigcap_{r \geq 0} \Pi\left(t, \Pi\left(r, \mathcal{B}_{s}\right)\right) \\
& =\bigcap_{r \geq 0} \Pi\left(t+r, \mathcal{B}_{s}\right)=\bigcap_{r \geq t} \Pi\left(r, \mathcal{B}_{s}\right)=\bigcap_{r \geq 0} \Pi\left(r, \mathcal{B}_{s}\right)=\mathcal{A}_{s}
\end{aligned}
$$

where we have used the semigroup property of $\Pi$ and the positive invariance of $\mathcal{B}_{s}$.

For the converse, $\mathcal{A}_{s} \subset \Pi\left(t, \mathcal{A}_{s}\right)$, pick $a \in \mathcal{A}_{s}$. Then, there exist sequences $\tau_{n} \rightarrow \infty$, and $a_{n} \in \Pi\left(\tau_{n}, \mathcal{B}_{s}\right)$ with $a_{n} \rightarrow a$. Consider $t>0$ and $n(t)$ such that for all $n \geq n(t), \tau_{n}-t \geq 0$. Then, for all $n \geq n(t)$ :

$$
a_{n} \in \Pi\left(\tau_{n}, \mathcal{B}_{s}\right)=\Pi\left(t, \Pi\left(\tau_{n}-t, \mathcal{B}_{s}\right)\right.
$$

and thus there exists a sequence $a_{n}^{\prime} \in \Pi\left(\tau_{n}-t, \mathcal{B}_{s}\right)$ with $a_{n} \in \Pi\left(t, a_{n}^{\prime}\right)$. Since $\mathcal{B}_{s}$ is compact and positively invariant, we deduce from

$$
a_{n}^{\prime} \in \Pi\left(\tau_{n}-t, \mathcal{B}_{s}\right) \subset \mathcal{B}_{s}
$$


the existence of a convergent subsequence $a_{n_{j}}^{\prime} \rightarrow a^{\prime} \in \mathcal{B}_{s}$ if $j \rightarrow \infty$. Of course, $\tau_{n_{j}}-t \rightarrow \infty$ and therefore, $a^{\prime} \in \mathcal{A}_{s}$. By the upper semicontinuity we have that

$$
H^{*}\left(\Pi\left(t, a_{n_{j}}^{\prime}\right), \Pi\left(t, a^{\prime}\right)\right) \rightarrow 0 \quad j \rightarrow \infty
$$

and from $a_{n_{j}} \rightarrow a$ and $a_{n_{j}} \in \Pi\left(t, a_{n_{j}}^{\prime}\right)$ we obtain $a \in \Pi\left(t, a^{\prime}\right) \subset \Pi\left(t, \mathcal{A}_{s}\right)$ as desired.

Since a compact set $K$ that is $\Pi$-invariant satisfies by the attraction property, $\operatorname{dist}\left(\Pi(t, K), \mathcal{A}_{s}\right) \rightarrow 0$, and we have that $\operatorname{dist}\left(K, \mathcal{A}_{s}\right)=\operatorname{dist}\left(\Pi(t, K), \mathcal{A}_{s}\right)$, then $\operatorname{dist}\left(K, \mathcal{A}_{s}\right)=0$ and so $K \subset \mathcal{A}_{s}$.

On the other hand, for a closed set $B$ attracting bounded sets, we have that $\operatorname{dist}\left(\mathcal{A}_{s}, B\right)=\operatorname{dist}\left(\Pi\left(t, \mathcal{A}_{s}\right), B\right) \rightarrow 0$ and therefore $\operatorname{dist}\left(\mathcal{A}_{s}, B\right)=0$ and $\mathcal{A}_{s} \subset B$.

Indeed, for the last statement, it is enough to prove that $\mathcal{A}_{s}$ attracts a connected bounded set $\tilde{\mathcal{B}}$ containing $\mathcal{A}_{s}$, which is trivial here by taking $\tilde{\mathcal{B}}=B\left(0,\left\|\mathcal{B}_{s}\right\|\right) \supset$ $\mathcal{B}_{s} \supset \mathcal{A}_{s}$. Suppose by contradiction that $\mathcal{A}_{s}$ is not connected, then there exists two disjoint open sets $O_{i}(\mathrm{i}=1,2)$ with $\mathcal{A}_{s} \subset O_{1} \cup O_{2}$ and $\mathcal{A}_{s} \cap O_{i} \neq \varnothing$. Denote by $\omega_{i}=\mathcal{A}_{s} \cap O_{i}$, which are closed, and therefore compact sets. Take $\epsilon>0$ such that $B\left(\omega_{1}, \epsilon\right) \cap B\left(\omega_{2}, \epsilon\right)=\varnothing$. By the attraction property, there exists $t_{\tilde{\mathcal{B}}}$ with $\Pi\left(t_{\tilde{\mathcal{B}}}, \tilde{\mathcal{B}}\right) \subset B\left(\mathcal{A}_{s}, \epsilon\right)$. But $\Pi\left(t_{\tilde{\mathcal{B}}}, \cdot\right)$ is u.s.c. and $\tilde{\mathcal{B}}$ is connected, and so it is $\Pi\left(t_{\tilde{\mathcal{B}}}, \tilde{\mathcal{B}}\right)$. Therefore, it can only be contained in one of the sets $B\left(\omega_{i}, \epsilon\right)$, which contradicts the inclusions

$$
\mathcal{A}_{s} \subset \Pi\left(t_{\tilde{\mathcal{B}}}, \mathcal{A}_{s}\right) \subset \Pi\left(t_{\tilde{\mathcal{B}}}, \tilde{\mathcal{B}}\right)
$$

\subsection{Autonomous weak attractors of SVSDS. Proof of Theorem 9}

Nonempty and compact: consider any sequences $\tau_{n} \rightarrow \infty$ and $b_{n} \in \mathcal{B}_{w}$. By the weak positive invariance of $\mathcal{B}_{w}$, there exist trajectories $\pi_{n}$ with $\pi_{n}(0)=b_{n}$ and $\pi_{n}(t) \in \mathcal{B}_{w}$ for all $t \geq 0$. In particular, $a_{n}=\pi_{n}\left(\tau_{n}\right) \in \mathcal{B}_{w}$, and by the compactness of $\mathcal{B}_{w}$ we can extract a subsequence $a_{n_{j}}$ converging to an element $a$ in $\mathcal{B}_{w}$ as $j \rightarrow \infty$. Taking $\left\{\tau_{n_{j}}, b_{n_{j}}, a_{n_{j}}\right\}_{j}$ as the original sequences, we have that $a \in \mathcal{A}_{w}$ which is therefore nonempty.

To show that $\mathcal{A}_{w}$ is compact, we only need to see that it is closed since is contained in the compact set $\mathcal{B}_{w}$. Suppose $a_{k} \in \mathcal{A}_{w}$ and $a_{k} \rightarrow a$ as $k \rightarrow \infty$. Then, there exist sequences $\tau_{k, n} \rightarrow \infty$ as $n \rightarrow \infty$ and trajectories $\pi_{k, n}$ with $\pi_{k, n}(t) \in \mathcal{B}_{w}$ for all $t \geq 0$ and $\pi_{k, n}\left(\tau_{k, n}\right) \rightarrow a_{k}$ as $n \rightarrow \infty$. Pick $n_{k}$ so that

$$
\left|\pi_{k, n_{k}}\left(\tau_{k, n_{k}}\right)-a_{k}\right| \leq 1 / k \quad \text { and } \quad t_{k+1, n_{k+1}} \geq t_{k, n_{k}}+1 \quad \forall k \in \mathbb{Z}^{+} .
$$

Then

$\left|\pi_{k, n_{k}}\left(\tau_{k, n_{k}}\right)-a\right| \leq\left|\pi_{k, n_{k}}\left(\tau_{k, n_{k}}\right)-a_{k}\right|+\left|a_{k}-a\right| \leq 1 / k+\left|a_{k}-a\right| \rightarrow 0 \quad$ as $\quad k \rightarrow \infty$.

Taking $\left\{\pi_{k, n_{k}}, \tau_{k, n_{k}}\right\}_{k}$ as the original sequences, we have again that $a \in \mathcal{A}_{w}$, which is closed as desired, and hence compact.

Weak positive invariance: Take $a \in \mathcal{A}_{w}$, then there exist a sequence $\tau_{n} \rightarrow \infty$ and trajectories $\pi_{n}$ with $\pi(t) \in \mathcal{B}_{w}$ for all $t \geq 0$, such that $\pi_{n}\left(\tau_{n}\right) \rightarrow a$ as $n \rightarrow \infty$. If we denote $v_{n}(t):=\pi_{n}\left(\tau_{n}+t\right)$, it is obvious that $v_{n}$ is a trajectory and $v_{n}(t) \in \mathcal{B}_{w}$ for 
all $t \geq 0$ and $v_{n}(0) \rightarrow a \in \mathcal{A}_{w}$. Applying Barbashin's theorem [20,13] on an interval, say $[0, T]$, we obtain a convergent subsequence $v_{n_{j}}(t) \rightarrow v(t)$ uniformly for $t \in[0, T]$. Naturally, $v:[0, T] \rightarrow \mathcal{B}_{w}$ is a trajectory and $v(0)=a$. Moreover, $v(t) \in \mathcal{A}_{w}$ since $\pi_{n_{j}}\left(\tau_{n_{j}}\right) \in \mathcal{B}_{w}, \pi_{n_{j}}\left(\tau_{n_{j}}+t\right) \rightarrow v(t)$ and $\tau_{n_{j}}+t \rightarrow \infty$. A Cantor diagonal argument shows again that we can obtain a trajectory defined on all of $\mathbb{R}^{+}$.

Weak negative invariance: The same idea can be used backwards in time. For any $T>0$, consider $n_{T}$ such that $\tau_{n}-T \geq 0$ for all $n \geq n_{T}$, and write

$$
v_{n}:[-T, 0] \rightarrow \mathcal{B}_{w}: s \mapsto v_{n}(s):=\pi_{n}\left(\tau_{n}+s\right) .
$$

Barbashin's theorem can be applied successively on intervals $[-T, 0],[-2 T,-T], \ldots$ and by a diagonal argument we obtain the existence of a trajectory $\bar{v}: \mathbb{R}^{-} \rightarrow \mathcal{B}_{w}$, which indeed takes values in $\mathcal{A}_{w}$ as before, with $\bar{v}(0)=a$. The concatenation of $v$ and $\bar{v}$ gives us the invariance of $\mathcal{A}_{w}$ as desired.

Weak attraction: Let $D$ be a bounded subset of $\mathbb{R}^{d}$. Since $\mathcal{B}_{w}$ is weakly absorbing, there exists a time $T_{D}>0$ such that for each $d_{n} \in D$ there exists a trajectory $\pi_{n}$ with $\pi_{n}(0)=d_{n}$ and $\pi_{n}(t) \in \mathcal{B}_{w}$ for all $t \geq T_{D}$.

By the weak positive invariance of $\mathcal{B}_{w}$, we can consider trajectories $\tilde{\pi}_{n}: \mathbb{R}^{+} \rightarrow \mathcal{B}_{w}$ with $\tilde{\pi}_{n}(0)=\pi_{n}\left(T_{D}\right)$. Since $\mathcal{B}_{w}$ is compact, for any sequence $\tau_{n, k} \rightarrow \infty$ as $k \rightarrow \infty$, there exist subsequences $\tilde{\pi}_{n}\left(\tau_{n, k^{\prime}}\right) \rightarrow a_{n}$ as $k^{\prime} \rightarrow \infty$ for some $a_{n} \in \mathcal{B}_{w}$ (for each $n$ ). By definition of $\mathcal{A}$, we have that $a_{n} \in \mathcal{A}_{w}$. Define

$$
\pi_{n}^{*}(t):= \begin{cases}\pi_{n}(t) & 0 \leq t \leq T_{D} \\ \tilde{\pi}_{n}\left(t-T_{D}\right) & t \geq T_{D}\end{cases}
$$

Then, $\pi_{n}^{*}(0)=d_{n}$ and $\pi_{n}^{*}\left(\tau_{n, k^{\prime}}+T_{D}\right) \rightarrow a_{n}$ as $k^{\prime} \rightarrow \infty$. Pick $k_{n}^{\prime}$ such that $\tau_{n, k_{n}^{\prime}}<$ $\tau_{n+1, k_{n+1}^{\prime}}$ and $\operatorname{dist}\left(\pi_{n}^{*}\left(\tau_{n, k_{n}^{\prime}}+T_{D}\right), \mathcal{A}_{w}\right) \leq 1 / n$. Therefore we have obtained for the trajectories $\pi_{n}^{*}$ which start at $d_{n}$ that

$$
\operatorname{dist}\left(\pi_{n}^{*}\left(\tau_{n, k_{n}^{\prime}}+T_{D}\right), \mathcal{A}_{w}\right) \rightarrow 0 .
$$

Thus, we have weak attraction.

The maximality statement w.r.t. $\mathcal{B}_{w}$ comes from its definition.

\subsection{Attractors for SVSPF and their restrictions}

\subsubsection{Proof of proposition 11}

The first assertion is obvious.

For the second one, as $P^{*}$ is the projection onto $P$ of the attractor $\mathcal{A}_{s}$, for every $p^{*} \in P^{*}, A_{s}\left(p^{*}\right)$ is nonempty. Compactness follows from that of $\mathcal{A}_{s}$ and the continuity of the projection of $P^{*} \times \mathbb{R}^{d}$ onto $\mathbb{R}^{d}$. The $\Phi$-invariance of $A_{s}(p)$ follows trivially from the $\Pi$-invariance of $\mathcal{A}_{s}$.

We now prove the third claim. Since $A_{s}(p)$ is compact, it is equivalent to prove $\varepsilon$-u.s.c. (cf. [1]). Suppose not, then there exists a constant $\varepsilon>0$ and $p_{n} \rightarrow$ 
$p$ (elements of $\left.P^{*}\right)$ such that $A_{s}\left(p_{n}\right) \not \subset B\left(A_{s}(p), \varepsilon\right)$, i.e. there exists a sequence $x_{n} \in A_{s}\left(p_{n}\right)$ with $x_{n} \notin B\left(A_{s}(p), \varepsilon\right)$. By the sectorial definition of $A_{s}(p)$, for each $\left(p_{n}, x_{n}\right)$, there exist sequences $t_{m}^{n} \rightarrow \infty$ as $m \rightarrow \infty$ and $y_{m}^{n} \in \Phi\left(t_{m}^{n}, p_{m}^{n}, b_{m}^{n}\right)$, with $\left(p_{m}^{n}, b_{m}^{n}\right) \subset \mathcal{B}_{s}$, such that $\left(\theta_{t_{m}^{n}} p_{m}^{n}, y_{m}^{n}\right) \rightarrow\left(p_{n}, x_{n}\right)$ as $m \rightarrow \infty$. Pick $m(n)$ strictly increasing such that $t_{m(n)}^{n}$ is also strictly increasing. From $\left(\theta_{t_{m(n)}^{n}} p_{m(n)}^{n}, y_{m(n)}^{n}\right)$ we can extract a subsequence converging to a pair $(p, y) \in\{p\} \times A_{s}(p)$, as they belong to $\mathcal{B}_{s}$ asymptotically. But this means that a subsequence of $x_{n}$ approximates $y \in A_{s}(p)$, which is a contradiction.

\subsubsection{Proof of Proposition 12}

Obviously $\mathcal{A}_{s}^{*} \subset \mathcal{A}_{s}$, since $\mathcal{A}_{s}^{*}$ attracts a smaller class of sets, in fact, just those from $P^{*} \times \mathbb{R}^{d}$ rather than $P \times \mathbb{R}^{d}$.

Now $\Pi^{*}\left(t, \mathcal{A}_{s}\right) \equiv \Pi\left(t, \mathcal{A}_{s}\right) \equiv \mathcal{A}_{s}, \forall t \geq 0$ since $\mathcal{A}_{s} \subset P^{*} \times \mathbb{R}^{d}$ and $\Pi^{*} \equiv \Pi$ on $P^{*} \times \mathbb{R}^{d}$. Since $\mathcal{A}_{s}^{*}$ attracts nonempty bounded subsets of $P^{*} \times \mathbb{R}^{d}$ including $\mathcal{A}_{s}$, one has that

$$
H^{*}\left(\mathcal{A}_{s}, \mathcal{A}_{s}^{*}\right)=H^{*}\left(\Pi^{*}\left(t, \mathcal{A}_{s}\right), \mathcal{A}_{s}^{*}\right) \rightarrow 0, \text { as } t \rightarrow \infty,
$$

i.e. $H^{*}\left(\mathcal{A}_{s}, \mathcal{A}_{s}^{*}\right) \equiv 0$, thus $\mathcal{A} \subset \mathcal{A}^{*}$ and therefore $\mathcal{A}_{s} \equiv \mathcal{A}_{s}^{*}$.

Alternatively, one can also argue in the following way: $\mathcal{A}_{s}$ is a $\Pi^{*}$-invariant set and the global attractor $\mathcal{A}_{s}^{*}$ of $\Pi^{*}$ in $P^{*} \times \mathbb{R}^{d}$ is the maximal compact $\Pi^{*}$-invariant subset of $P^{*} \times \mathbb{R}^{d}$, so $\mathcal{A}_{s} \subset \mathcal{A}_{s}^{*}$.

\subsubsection{Proof of proposition 13}

The statement about $P^{*}$ is clear even in this weak framework since $\theta$ is single-valued and the weak $\Pi$-invariance easily implies $\theta$-invariance for $P^{*}$, and the weak attraction property of $\mathcal{A}_{w}$ implies the strong attraction property for $\theta$ in $P^{*}$.

That $A_{w}(p)$ is nonempty is trivial, as mentioned before, since $P^{*}$ is the projection of $\mathcal{A}_{w}$ onto $P$. It is also compact, the proof is the same in Theorem 9 , since for all $a_{k} \in P \times \mathbb{R}^{d}$ we have that $\operatorname{Pr}_{P}\left(a_{k}\right)=p$ and so the limit $a$.

Let us prove that $P^{*} \ni p \mapsto A_{w}(p)$ is u.s.c. We want to see that if $p^{\prime} \rightarrow p$, then $H^{*}\left(A_{w}\left(p^{\prime}\right), A_{w}(p)\right) \rightarrow 0$. If not, there exist a constant $\varepsilon>0$ and a sequence $p_{n} \rightarrow p$ with $\varepsilon \leq H^{*}\left(A_{w}\left(p_{n}\right), A_{w}(p)\right)=\operatorname{dist}\left(a_{n}, A_{w}(p)\right)$, where we have used that $A_{w}\left(p_{n}\right)$ is compact. Therefore,

$$
\varepsilon \leq \operatorname{dist}\left(a_{n}, a\right) \quad \forall a \in A_{w}(p) .
$$

As $A_{w}\left(p_{n}\right) \subset \operatorname{Pr}_{\mathbb{R}^{d}} \mathcal{A}_{w}$, which is compact, from $\left\{a_{n}\right\}$ we extract a convergent subsequence (which we do not relabel), and so $\left(p_{n}, a_{n}\right) \rightarrow(p, a)$. From the weak invariance of $\mathcal{A}_{w}$, there exists at least one entire trajectory $\pi_{n}$ passing through each $\left(p_{n}, a_{n}\right)$. Barbashin's theorem (Th. 2, see Remark 5) provides a converging subsequence $\left\{\pi_{n_{1}}\right\}_{n_{1}}$ on the interval $[-1,1]$ to a trajectory. Applying it again to this subsequence we obtain another one denoted by $\left\{\pi_{n_{2}}\right\}_{n_{2}}$, which is uniformly converging on $[-2,2]$. A diagonal argument gives an entire trajectory $\pi$ such that $\pi(0)=(p, a)$. By Remark 10, we have that $(p, a) \in \mathcal{A}_{w}$, so $a \in A_{w}(p)$, which contradicts (10).

The last statement is obvious. 


\subsection{Pullback structure of SVSPF attractors}

\subsubsection{Proof of Lemma 15}

Thanks to the $\Phi$-positive invariance of $B_{s}(p)$ for $p \in P^{*}$, and the cocycle property, we have that

$$
\begin{aligned}
\Phi\left(t+r, \theta_{-t-r} p, B_{s}\left(\theta_{-t-r} p\right)\right) & =\Phi\left(t, \theta_{-t} p, \Phi\left(r, \theta_{-t-r} p, B_{s}\left(\theta_{-t-r} p\right)\right)\right) \\
& \subset \Phi\left(t, \theta_{-t} p, B_{s}\left(\theta_{-t} p\right)\right) .
\end{aligned}
$$

Thus, these sets are nested, and by the u.s.c. of $\Phi$ on its third variable and the

compactness of $B_{s}(p)$ for $p \in P^{*}$, they are compact. Thus, $\hat{A}_{s}(p)$ is nonempty and compact.

\subsubsection{Proof of Proposition 17}

We only need to check the first identity since the second one has already been proved.

First, let us prove that $\hat{A}_{s}(p) \subset A_{s}^{*}(p)$ :

$$
\begin{aligned}
\{p\} \times \hat{A}_{s}(p) & =\{p\} \times \bigcap_{t \geq 0} \Phi\left(t, \theta_{-t} p, B_{s}\left(\theta_{-t} p\right)\right) \\
& =\bigcap_{t \geq 0}\{p\} \times \Phi\left(t, \theta_{-t} p, B_{s}\left(\theta_{-t} p\right)\right) \\
& =\bigcap_{t \geq 0}\left\{\theta_{t}\left(\theta_{-t} p\right)\right\} \times \Phi\left(t, \theta_{-t} p, B_{s}\left(\theta_{-t} p\right)\right) \\
& =\bigcap_{t \geq 0} \Pi\left(t,\left(\theta_{-t} p, B_{s}\left(\theta_{-t} p\right)\right)\right) \\
& \subset \bigcap_{t \geq 0} \Pi\left(t, \mathcal{B}_{s}^{*}\right) \equiv \mathcal{A}_{s}^{*} .
\end{aligned}
$$

Here we have used that $P^{*}$ is $\theta$-invariant. Thus $\{p\} \times \hat{A}_{s}(p) \subset \mathcal{A}_{s}^{*}$ what implies that $\{p\} \times \hat{A}_{s}(p) \subset\{p\} \times A_{s}^{*}(p)$ and therefore $\hat{A}_{s}(p) \subset A_{s}^{*}(p)$ as desired.

As for the converse, notice that $\Pi^{*}\left(t, \mathcal{A}_{s}^{*}\right)=\mathcal{A}_{s}^{*}$ for all $t \geq 0$. So, in particular,

$$
\Phi\left(t, p, A_{s}^{*}(p)\right)=A_{s}^{*}\left(\theta_{t} p\right) \quad \text { for all } \quad t \geq 0, p \in P^{*} .
$$

Moreover, we know that

$$
\hat{A}_{s}(p) \subset A_{s}^{*}(p) \subset B_{s}(p) .
$$

Thus, setting $\theta_{-t} p$ instead of $p$,

$$
A_{s}^{*}(p)=\Phi\left(t, p, A_{s}^{*}\left(\theta_{-t} p\right)\right) \subset \Phi\left(t, \theta_{-t} p, B_{s}\left(\theta_{-t} p\right)\right)
$$

for all $t \geq 0$ and $p \in P^{*}$. Therefore, we finally obtain that

$$
A_{s}^{*}(p) \subset \bigcap_{t \geq 0} \Phi\left(t, \theta_{-t} p, B_{s}\left(\theta_{-t} p\right)\right)=\hat{A}_{s}(p) .
$$




\subsubsection{Proof of Proposition 18}

Suppose not, then there exist a positive constant $\varepsilon$, a bounded set $D$ and sequences $t_{n} \rightarrow \infty, y_{n} \in \Phi\left(t_{n}, \theta_{-t_{n}} p, d_{n}\right)$ with $p \in P^{*}$ and $d_{n} \in D$ such that

$$
\operatorname{dist}\left(y_{n}, \hat{A}_{s}(p)\right)>\varepsilon>0 .
$$

There exists $T_{D}(p)$ such that $\Phi\left(t, \theta_{-t} p, D\right) \subset B_{s}(p)$ for all $t \geq T_{D}(p)$. So, as $B_{s}(p)$ is compact, there exists a converging subsequence (denoted the same) $y_{n} \rightarrow x \in B_{s}(p)$. We will see that in fact $x \in \hat{A}_{s}(p)$, which will be a contradiction.

Consider any $\tau>0$ and take $n(\tau)$ big enough such that $t_{n}-\tau>0$ and $\Phi\left(t_{n}-\right.$ $\left.\tau, \theta_{-t_{n}} p, d_{n}\right) \subset B_{s}\left(\theta_{-\tau} p\right)$ which is possible since $t_{n} \rightarrow \infty$ and the family $B_{s}(p)$ is $\Phi$-pullback absorbing.

Then, we have that

$$
y_{n} \in \Phi\left(t_{n}, \theta_{-t_{n}} p, d_{n}\right)=\Phi\left(\tau, \theta_{-\tau} p, \Phi\left(t_{n}-\tau, \theta_{-t_{n}} p, d_{n}\right)\right),
$$

which shows that $x \in \hat{A}_{s}(p)$ as desired.

Now we show that this is the minimal pullback attractor, i.e. it coincides with the closure of the union of the omega-limit sets on each $p$-fiber:

$$
\bigcup_{D \text { bounded }} \bigcap_{\tau \geq 0} \overline{\bigcup_{t \geq \tau} \Phi\left(t, \theta_{-t} p, D\right)} .
$$

Indeed, this is a trivial consequence of the fact that for all $p \in P^{*}, B_{s}(p)$ is contained in a compact set $K$, namely, $K=\operatorname{Pr}_{\mathbb{R}^{d}} \mathcal{B}_{s}$ :

$$
\Phi\left(t, \theta_{-t} p, B_{s}\left(\theta_{-t} p\right)\right) \subset \Phi\left(t, \theta_{-t} p, K\right)
$$

and so

$$
\hat{A}_{s}(p) \subset \overline{\bigcup_{\text {bounded }} \bigcap_{\tau \geq 0} \overline{\bigcup_{t \geq \tau} \Phi\left(t, \theta_{-t} p, D\right)}}
$$

The other inclusion is obvious, using the minimality of the omega-limit set for $D$ at fiber $p$.

Acknowledgments P.M-R thanks all the staff of the Fachbereich Mathematik of the J. W. Goethe Universität, and specially to Professor P. E. Kloeden, for their kind hospitality during his visit preparing this work.

This work was partially supported by the DAAD (Germany), the Ministerio de Ciencia y Tecnología (Spain) under the projects HA2001-0075 and BFM2002-03068.

\section{References}

[1] J. P. Aubin and A. Cellina, Differential Inclusions. Set-Valued Maps and Viability Theory, Springer-Verlag, Berlin, 1984.

[2] J. P. Aubin and H. Frankowska, Set-Valued Analysis, Birkhäuser, Boston, 1990. 
[3] E. A. Barbashin, On the theory of generalized dynamical systems, Uch. Zap. Moskov. Gos. Univ. 135 (1949), 110-133.

[4] T. Caraballo, J. A. Langa, and J. Valero, Global attractors for multivalued random dynamical systems, Nonlinear Anal. 48 (2002), no. 6, 805-829.

[5] T. Caraballo, P. Marín-Rubio, and P. E. Kloeden, Weak pullback attractors of setvalued processes, Submitted.

[6] D. N. Cheban, Global attractors of infinite-dimensional nonautonomous dynamical systems I, Bull. Acad. Sciences Rep. Moldova. Mat. 25 (1997), no. 3, $42-55$.

[7] _ Global attractors of infinite-dimensional nonautonomous dynamical systems II, Bull. Acad. Sciences Rep. Moldova. Mat. 27 (1998), no. 2, 25-38, $132,135$.

[8] D. N. Cheban, P. E. Kloeden, and B. Schmalfuß, The relationship between pullback, forwards and global attractors of nonautonomous dynamical systems, Nonlinear Dynamics \& Systems Theory 2 (2002), no. 2, 9-28.

[9] D. N. Cheban and B. Schmalfuß, Global attractors of nonautonomous disperse dynamical systems and differential inclusions, Bull. Acad. Sciences Rep. Moldova. Mat. 29 (1999), no. 1, 3-22.

[10] H. Crauel, A. Debussche, and F. Flandoli, Random attractors, J. Dyn. Diff. Eqns. 9 (1997), no. 2, 307-341.

[11] H. Crauel and F. Flandoli, Attractors for random dynamical systems, Probab. Theory Relat. Fields 100 (1994), no. 3, 365-393.

[12] R. Johnson and M. Nerurkar, Controllability, Stabilization, and the Regulator Problem for Random Differential Systems, vol. 136, Memoirs of the American Mathematical Society, no. 646, Amer. Math. Soc., Providence, Rhode Island, 1998.

[13] P. E. Kloeden, General control systems without backwards extension, Differential Games and Control Theory (P. Liu E. Roxin and R. Sternberg, eds.), MarcelDekker, 1974, pp. 49-58.

[14]_ General control systems, Mathematical Control Theory (W. A. Coppel, ed.), Lecture Notes in Mathematics, vol. 680, Springer-Verlag, 1978, pp. 119 138.

[15] _ Pullback attractors in nonautonomous difference equations, J. Difference Eqns. Applns. 6 (2000), 33-52.

[16] P. E. Kloeden and P. Marín-Rubio, Weak pullback attractors in nonautonomous difference inclusions, J. Difference Eqns. Applns., To appear. 
[17] P. E. Kloeden and B. Schmalfuß, Nonautonomous systems, cocycle attractors and variable time-step discretization, Numer. Algorithms 14 (1997), no. 1-3, $141-152$.

[18] V. S. Melnik and J. Valero, On attractors of multivalued semi-flows and differential inclusions, Set-Valued Anal. 6 (1998), no. 1, 83-111.

[19] E. O. Roxin, On generalized dynamical systems defined by contingent equations, J. Diff. Eqns. 1 (1965), 188-205.

[20] _ Stability in general control systems, J. Diff. Eqns. 1 (1965), 115-150.

[21] B. Schmalfuß, Backward cocycles and attractors of stochastic differential equations, International Seminar on Applied Mathematics-Nonlinear Dynamics: Attractor Approximation and Global Behaviour (Dresden) (V. Reitmann, T. Redrich, and N. J. Kosch, eds.), Technische Universität, 1992, pp. 185-192.

[22] G. Sell, Non-autonomous differential equations and dynamical systems, Amer. Math. Soc. 127 (1967), 241-283.

[23] G. V. Smirnov, Introduction to the Theory of Differential Inclusions, Amer. Math. Soc., Providence, 2002.

[24] G. P. Szegö and G. Treccani, Semigruppi di Trasformazioni Multivoche, Springer Lecture Notes in Mathematics, vol. 101, Springer-Verlag, 1969. 